\title{
Persistence of antimicrobial resistance in respiratory streptococci
}

\author{
Robin Bruyndonckx ${ }^{\mathrm{a}, \mathrm{b}, *}$, Niel Hens ${ }^{\mathrm{a}, \mathrm{c}}$, Marc Aerts $^{\mathrm{a}}$, Herman Goossens ${ }^{\mathrm{b}}$, Katrien Latour ${ }^{\mathrm{d}}$, \\ Boudewijn Catry ${ }^{\mathrm{d}}$, Samuel Coenen ${ }^{\mathrm{b}, \mathrm{e}}$ \\ a Interuniversity Institute for Biostatistics and Statistical Bioinformatics (I-BIOSTAT), University of Hasselt, Agoralaan 1, Hasselt, Belgium \\ ${ }^{\mathrm{b}}$ Laboratory of Medical Microbiology, Vaccine E Infectious Disease Institute (VAXINFECTIO), University of Antwerp, Universiteitsplein 1, Wilrijk, Belgium \\ ${ }^{\mathrm{c}}$ Centre for Health Economic Research and Modelling Infectious Diseases (CHERMID), Vaccine E' Infectious Disease Institute (VAXINFECTIO), University of \\ Antwerp, Universiteitsplein 1, Wilrijk, Belgium \\ ${ }^{\mathrm{d}}$ Scientific Institute of Public Health (WIV-ISP), Juliette Wytsmanstraat 14, Brussels, Belgium \\ e Centre for General Practice, Vaccine E'Infectious Disease Institute (VAXINFECTIO), University of Antwerp, Universiteitsplein 1, Wilrijk, Belgium
}

\section{A R T I C L E I N F O}

\section{Article history:}

Received 4 April 2016

Received in revised form 16 August 2016

Accepted 24 September 2016

Available online 9 November 2016

\section{Keywords:}

Antimicrobial resistance

Baseline resistance

Generalised estimating equation

Streptococcus pneumoniae

Streptococcus pyogenes

\begin{abstract}
A B S T R A C T
Objectives: To assess whether persistence of antimicrobial resistance (i.e. non-susceptible resistance status) after treatment with penicillins or cephalosporins versus macrolides or tetracyclines differs and to compare the results obtained using routinely collected data with findings reported in prospective studies.

Methods: Routinely collected microbiological data from 14 voluntary participating laboratories (2005) containing information on resistance status and individual antimicrobial consumption patterns (mid 2004-2005) were analysed using a generalised estimating equation (GEE) approach. The link function was adjusted to acknowledge that the proportion of resistant isolates in the population not treated with antibiotics [baseline resistance (BR)] is not necessarily zero. To optimise the comparability of this study with prospective studies, the analysis was repeated after removal of 14 isolates from patients who did not survive 2005.

Results: BR estimates were unstable and their confidence intervals were wide, which called for a sensitivity analysis using an adjusted GEE model with three different BR estimates. All models indicated that the proportion of susceptible isolates differed by treatment group and increased significantly over time, with this increase being independent of treatment group. Persistence of resistance after exposure to macrolides or tetracyclines was approximately three times as long as after exposure to penicillins or cephalosporins.

Conclusions: Resistance following treatment with macrolides or tetracyclines persists longer than following treatment with penicillins or cephalosporins, which confirms the findings from prospective studies and suggests the use of routinely collected data as a valuable alternative to determine such differences in persistence of resistance.

(c) 2016 International Society for Chemotherapy of Infection and Cancer. Published by Elsevier Ltd. All rights reserved.
\end{abstract}

\section{Introduction}

Antimicrobial resistance poses a substantial threat to public health as it is related to treatment failure, prolonged hospitalisation, increased cost of care and increased mortality [1-4]. Both ecological studies and randomised controlled trials in individual patients have demonstrated a link between antibiotic use and resistance [5-7]. Assessment of the impact of antibiotic use on

\footnotetext{
* Corresponding author at: Interuniversity Institute for Biostatistics and Statistical Bioinformatics (I-BIOSTAT), University of Hasselt, Agoralaan 1, Hasselt, Belgium. Fax: +32 11268299 .

E-mail address: robin.bruyndonckx@uhasselt.be (R. Bruyndonckx).
}

resistance in oropharyngeal streptococci showed that persistence of resistance (i.e. non-susceptible resistance status) after exposure to macrolides lasts for $>6$ months, whereas it is estimated to be much shorter after exposure to penicillins $[7,8]$.

Gaining a thorough understanding of the persistence of resistance for different combinations of bacteria and antibiotics would require a huge number of studies. Therefore, in this study we set out to assess whether routinely collected data on resistance and antibiotic use at the level of the individual patient confirm the conclusions reached in the prospective studies conducted by Malhotra-Kumar et al. [7] and Chung et al. [8] and hence could serve as a proxy to study other drug-bug combinations. 


\section{Methods}

\subsection{Data}

Data regarding information on the resistance status of isolates from individual patient samples retrieved from 14 voluntary participating laboratories (2005) as well as linked individual antimicrobial consumption data retrieved from the Belgian Intermutualistic Agency, which bundles national reimbursement information (July 2004-December 2005), were collected within a large case-control study assessing the link between antimicrobial consumption and resistance for patients in ambulatory care or nursing homes [9].

This study focused on the resistance status (i.e. susceptible versus non-susceptible) of respiratory Streptococcus pyogenes (PY) and Streptococcus pneumoniae (PN) isolates to penicillin and erythromycin following oral consumption of penicillins or cephalosporins [CD: substances with Anatomic Therapeutic Chemical (ATC) code J01C and J01D, respectively] and macrolides or tetracyclines (AF: substances with ATC code J01F and J01A, respectively) [10]. Because resistance to penicillin and erythromycin involves different mechanisms, we did not study penicillin resistance after treatment with $\mathrm{AF}$ or erythromycin resistance after treatment with $C D[11,12]$. Other explanatory variables that were considered to explain an isolate's resistance status were the sampling location (upper respiratory tract, ear, lower respiratory tract or sputum), sex (male or female), preferential reimbursement (yes or no), $\log$ (time), age, weight, number of prescription days (for antibiotics in the same treatment group), total dose prescribed (for antibiotics in the same treatment group), whether the patient survived the study (yes or no) and whether the patient was a child (defined as an individual aged $<12$ years, yes or no). $\log$ (time) was included as a potential explanatory variable rather than time because we assume that the time until the isolate tests susceptible follows a log-logistic distribution.

For each isolate, we considered the oral consumption of an antibiotic that was purchased from a pharmacy prior to sampling. Isolates for which the time between consumption and sampling was $\geq 4$ days ( $95 \%$ of isolates) were included to ensure that patients started taking the purchased antibiotic. The final data used in this paper contain information on resistance status for 451 isolates retrieved from 363 patients (Tables 1 and 2).

\subsection{Persistence of resistance}

A multiple logistic regression model was included using resistance status as binary outcome and a logit link function. Because the variables considered to explain antimicrobial resistance status were numerous (12 variables and all two-way interactions), an automatic forward selection procedure was used to reach a model including all significant explanatory variables and two-way interactions. The significance level for this procedure was set at 0.15 to avoid being overly conservative during this intermediate stage of model building.

Because multiple samples from the same patient were potentially taken, measurements obtained from the same patient

Table 1

Number of isolates per patient.

\begin{tabular}{ll}
\hline No. of isolates & No. of patients \\
\hline 1 & 288 \\
2 & 67 \\
3 & 5 \\
4 & 2 \\
6 & 1 \\
\hline
\end{tabular}

Table 2

Resistance status for Streptococcus pneumoniae (PN) and Streptococcus pyogenes (PY) isolates in the final data set tested following exposure to penicillins and cephalosporins (CD) or macrolides or tetracyclines (AF).

\begin{tabular}{llll}
\hline Resistance status & Bacteria & Treatment & No. of isolates \\
\hline Susceptible & PN & CD & 150 \\
& & AF & 33 \\
& PY & CD & 148 \\
& & AF & 37 \\
Non-susceptible & PN & CD & 38 \\
& & AF & 26 \\
& PY & CD & 6 \\
& & AF & 13 \\
\hline
\end{tabular}

were expected to be correlated. Ignoring correlation would typically result in underestimation of the standard errors and hence wrongfully retaining variables in the model $[13,14]$. Therefore, a generalised estimating equation (GEE) model [15] was constructed using an independent working correlation and explanatory variables that were retained in the automatic forward selection procedure (i.e. treatment, bacteria, survival status, log (time), interaction between survival status and $\log$ (time) and interaction between treatment and bacteria). The final model was obtained by using backward selection (significance level 0.05). Note that although this working correlation might be incorrect, parameter estimates and empirical standard errors are deemed consistent due to the use of a sandwich estimator in the GEE approach [13].

The data used in this study contain information on 14 isolates (all S. pneumoniae) taken from patients who did not survive 2005. Because the studies conducted by Malhotra-Kumar et al. [7] and Chung et al. [8] reported no deaths, the aforementioned analyses were repeated after exclusion of these samples to optimise comparability.

\subsection{Baseline resistance $(B R)$}

Both the logistic regression model and the GEE model implicitly assume that the proportion of non-susceptible isolates in the population falls back to zero when the timespan between antibiotic consumption and sampling becomes large enough. Several authors, however, found a non-zero proportion of nonsusceptible isolates at baseline (i.e. BR) [16-18]. Therefore, we relaxed this assumption and adjusted the link function accordingly: $\operatorname{logitp})=\log p(1-p)) \rightarrow \log p(g-p))$, with $g=1-B R$ and $p=$ the proportion of susceptible individuals. We allowed this BR to differ by treatment (CD or AF) and by type of bacteria (PY or PN) to avoid wrongfully concluding significant differences between treatments and bacteria types that were actually caused by the difference in BR.

Because the proportion of non-susceptible isolates was substantially higher in samples from patients who did not survive 2005 compared with the full data ( 0.64 and 0.23 , respectively), the estimates for BR were recalculated after exclusion of these samples.

\section{Results}

\subsection{Baseline resistance}

Because estimates for BR in this specific setting were not available, BR was calculated as the proportion of non-susceptible samples within a sliding 6-month time frame between $t-186$ and $t$ (with $t=186,187, \ldots, 372$ ) and the evolution of this estimate for BR over time was studied (Fig. 1). Since the BR estimates did not 

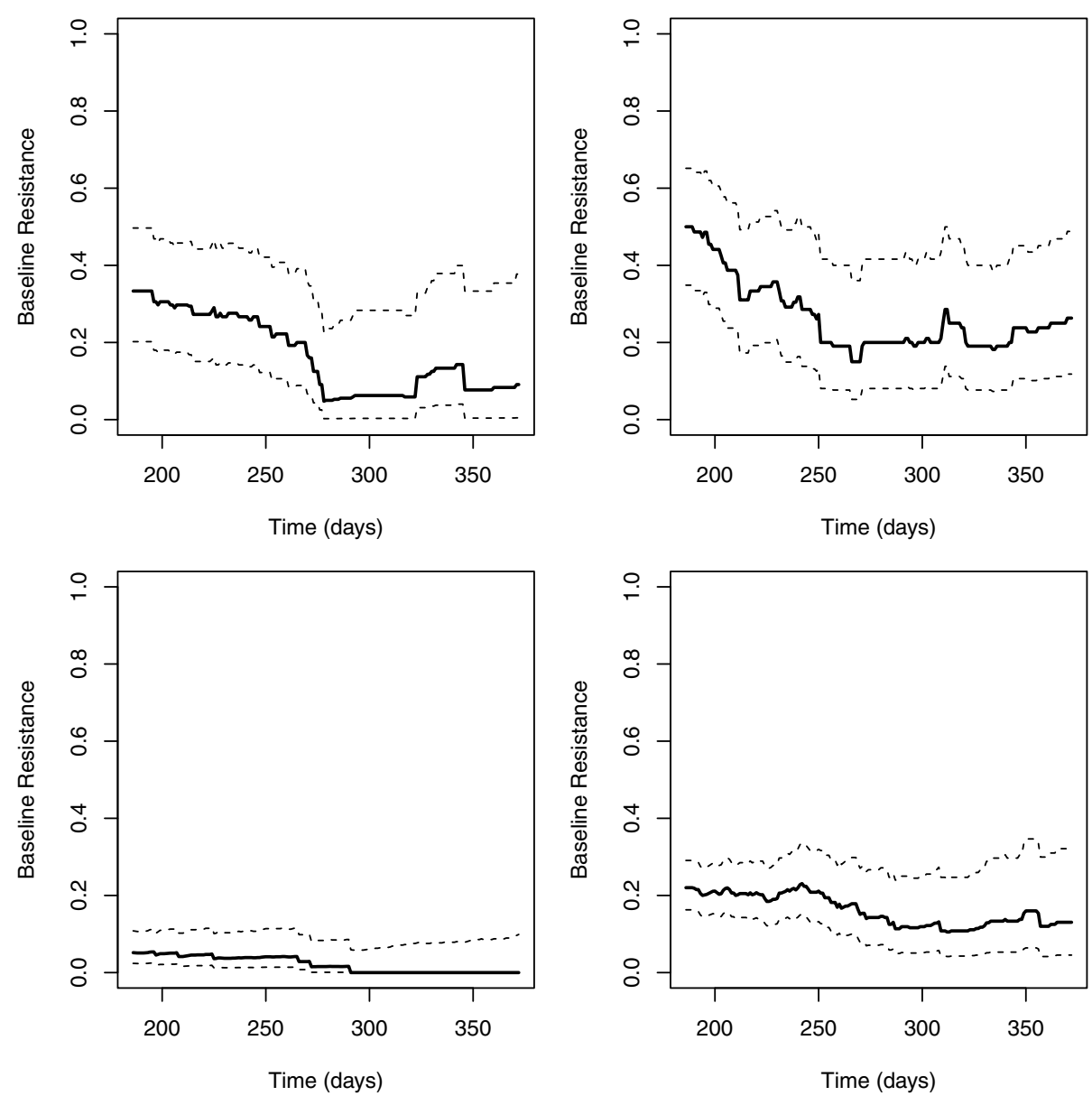

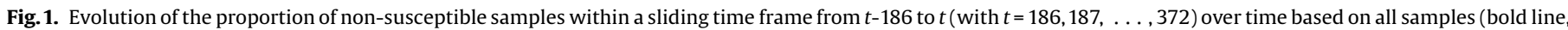

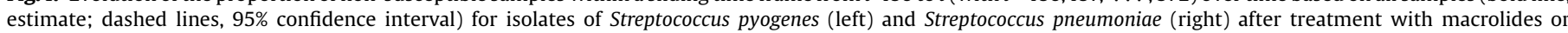
tetracyclines (top) and penicillins or cephalosporins (bottom).

stabilise, a sensitivity analysis was conducted in which the adjusted GEE model was fitted using different BR estimates [for $t=248$ (time frame 3-8 months, BR8), for $t=310$ (time frame 5-10 months, BR10) and for $t=372$ (time frame 7-12 months, BR12)] (Table 3). After dropping samples from patients who did not survive 2005, the BR estimates were recalculated (Table 4).

\subsection{Persistence of resistance}

From the unadjusted GEE model, we can conclude that the odds of being susceptible was significantly lower for treatment with $\mathrm{AF}$ and significantly higher for PY isolates (Table 5). After correcting for BR, parameter estimates changed and the odds of being susceptible no longer differed significantly by bacteria type. Parameter estimates differed when using different BR estimates, which stresses the need for the sensitivity analysis.
Further backward reduction (significance level 0.05) resulted in a model including the explanatory variables treatment, $\log$ (time) and survival status (Table 6). Based on the final model, we can conclude that the odds of being susceptible was significantly lower for treatment with AF whilst it was significantly higher when surviving 2005 and with increasing time since antibiotic consumption. The evolution of resistance over time, however, did not differ by treatment since including an interaction between log (time) and treatment in the final models resulted in $P$-values of 0.1147 (BR8), 0.3394 (BR10) and 0.3104 (BR12). These findings are illustrated in Fig. 2.

\subsection{Persistence of resistance for patients surviving 2005}

Fig. 2 shows that there is a difference between patients who did and those who did not survive 2005, which was found to be significant (Table 6). Because of this significant difference and

Table 3

Estimates (95\% confidence intervals) of baseline resistance (BR) based on all samples $(n=451)$.

\begin{tabular}{|c|c|c|c|}
\hline Bug-drug combination & BR8 & BR10 & BR12 \\
\hline PY-AF & $0.2414(0.1222-0.4211)$ & $0.0625(0.0032-0.2833)$ & $0.0909(0.0047-0.3774)$ \\
\hline PY-CD & $0.0400(0.0137-0.1111)$ & $0.0000(0.0000-0.0664)$ & $0.0000(0.0000-0.0989)$ \\
\hline $\mathrm{PN}-\mathrm{AF}$ & $0.2727(0.1315-0.4815)$ & $0.2500(0.1119-0.4687)$ & $0.2632(0.1181-0.4879)$ \\
\hline PN-CD & $0.2083(0.1305-0.3157)$ & $0.1081(0.0429-0.2471)$ & $0.1304(0.0454-0.3213)$ \\
\hline
\end{tabular}

PY, Streptococcus pyogenes; PN, Streptococcus pneumoniae; AF, treatment with macrolides or tetracyclines; CD, treatment with penicillins or cephalosporins.

a BR estimates for time frame 3-8 months (BR8), 5-10 months (BR10) and 7-12 months (BR12). 
Table 4

Estimates (95\% confidence intervals) of baseline resistance (BR) ${ }^{\mathrm{a}}$ based on samples from patients surviving 2005 ( $n=437$ ).

\begin{tabular}{|c|c|c|c|}
\hline Bug-drug combination & BR8 & BR10 & BR12 \\
\hline PY-AF & $0.2414(0.1222-0.4211)$ & $0.0625(0.0032-0.2833)$ & $0.0909(0.0047-0.3774)$ \\
\hline PY-CD & $0.0400(0.0137-0.1111)$ & $0.0000(0.0000-0.0664)$ & $0.0000(0.0000-0.0989)$ \\
\hline $\mathrm{PN}-\mathrm{AF}$ & $0.2727(0.1315-0.4815)$ & $0.2222(0.0900-0.4521)$ & $0.1875(0.0659-0.4301)$ \\
\hline $\mathrm{PN}-\mathrm{CD}$ & $0.1618(0.0928-0.2669)$ & $0.1081(0.0429-0.2471)$ & $0.1364(0.0475-0.3333)$ \\
\hline
\end{tabular}

PY, Streptococcus pyogenes; PN, Streptococcus pneumoniae; AF, treatment with macrolides or tetracyclines; $\mathrm{CD}$, treatment with penicillins or cephalosporins.

a BR estimates for time frame 3-8 months (BR8), 5-10 months (BR10) and 7-12 months (BR12).

Table 5

Parameter estimates for the generalised estimating equation (GEE) models on persistence of overall resistance using different estimates for baseline resistance $(\mathrm{BR})^{\mathrm{a}}$ obtained by forward model building.

\begin{tabular}{lcrrr}
\hline \multirow{2}{*}{ Parameter } & \multicolumn{3}{c}{ Parameter estimate } & \\
\cline { 2 - 5 } & \multicolumn{1}{c}{ BR $=0$} & \multicolumn{1}{c}{ BR8 } & \multicolumn{1}{c}{ BR10 } & \multicolumn{1}{c}{ BR12 } \\
\hline Intercept & 1.1015 & 1.0448 & 1.1622 & 1.1460 \\
Treatment AF & $-1.3204^{* *}$ & -2.3710 & $-1.3231^{* *}$ & $-1.4705^{* *}$ \\
Bacteria PY & $1.6892^{* *}$ & 2.9179 & 0.8955 & 0.6923 \\
Death no & -1.9274 & -3.5075 & -2.1480 & -2.0767 \\
Log(time) & -0.2928 & -0.0649 & -0.2409 & -0.2142 \\
Log(time) $\times$ death no & 0.8777 & 1.7286 & 1.0982 & 1.1159 \\
Treatment AF $\times$ bacteria PY & -1.1129 & -1.9666 & -1.0507 & -0.7902 \\
\hline
\end{tabular}

AF, treatment with macrolides or tetracyclines; PY, Streptococcus pyogenes.

${ }^{* * *} P<0.01$.

a BR estimates for time frame 3-8 months (BR8), 5-10 months (BR10) and 7-12 months (BR12).

Table 6

Parameter estimates for the generalised estimating equation (GEE) models on persistence of overall resistance using different estimates for baseline resistance $(B R)^{a}$ after final backward model reduction.

\begin{tabular}{llll}
\hline Parameter & \multicolumn{3}{l}{ Parameter estimate } \\
\cline { 2 - 4 } & BR8 & BR10 & BR12 \\
\hline Intercept & -2.4517 & -2.6359 & -2.6159 \\
Treatment AF & $-3.2705^{* *}$ & $-1.9071^{* *}$ & $-1.9512^{* *}$ \\
Death no & $3.3138^{*}$ & $2.5573^{* *}$ & $2.6152^{* *}$ \\
Log(time) & $0.9926^{*}$ & $0.7445^{* *}$ & $0.7613^{* *}$ \\
\hline
\end{tabular}

$\mathrm{AF}$, treatment with macrolides or tetracyclines; PY, Streptococcus pyogenes.

${ }^{*} P<0.05$.

*** $P<0.01$

a BR estimates for time frame 3-8 months (BR8), 5-10 months (BR10) and 712 months (BR12).

because the studies conducted by Malhotra-Kumar et al. [7] and Chung et al. [8] reported no deaths, the analysis was repeated on samples from patients who did survive 2005 to improve comparability.

From the unadjusted GEE model, we can conclude that the odds of being susceptible was significantly lower for treatment with AF whilst it was significantly higher for PY isolates and with increasing time between sampling and prescription (Table 7). After correcting for BR, parameter estimates changed and the odds of being susceptible no longer differed significantly by bacteria. Parameter estimates again differed when using different BR estimates, stressing the need for a sensitivity analysis.

Further backward reduction (significance level 0.05) resulted in a model including explanatory variables treatment and $\log$ (time) (Table 8). Based on the final models, we can conclude that the odds of being susceptible was significantly lower for treatment with AF whilst it was significantly higher with increasing time since antibiotic consumption. The evolution of resistance over time, however, did not differ by treatment since including an interaction between $\log$ (time) and treatment in the final models resulted in $P$ values of 0.1035 (BR8), 0.7040 (BR10) and 0.7095 (BR 12). These findings are illustrated in Fig. 3.
Fig. 3 also shows that the proportion of susceptible isolates stabilised more quickly after treatment with $C D$ than after treatment with AF. We considered the proportion of susceptible isolates to be stable when it increased by less than $0.05 \%$ per day. Table 9 demonstrates that resistance after treatment with $\mathrm{AF}$ persisted approximately three times as long as after treatment with CD.

\section{Discussion}

In this study, we aimed to assess whether routinely collected data on antibiotic use and resistance at the level of the individual patient can confirm the conclusions reached in prospective studies, which have shown that persistence of resistance following exposure to macrolides lasts for $>6$ months, whilst it is estimated to be much shorter after exposure to penicillins $[7,8]$. Use of routinely collected data is both a strength, as individuals were not exposed to an additional intervention (e.g. new treatment or placebo control), and a limitation of the study, as we had no control over prescribed dose, duration of treatment or treatment adherence. Major advantages of studying routinely collected data are that real field conditions are met and that it is less labour intensive and expensive than conducting a prospective study for each drugbug combination. Ethical and insurance concerns are also of another dimension given the retrospective concept of analysing routinely collected data. This analysis would, however, benefit from including more samples, which would make BR estimates more reliable and might make a sensitivity analysis redundant.

In the GEE analysis, we acknowledged that the proportion of non-susceptible samples taken from a population not treated with antibiotics does not necessarily equal zero and adjusted the model to account for a BR level. Because BR exists for most, if not all, antimicrobials, this adjusted GEE analysis should be the method of choice when analysing persistence of resistance of antimicrobials (both oral and parenteral). Here, BR was calculated as the proportion of non-susceptible isolates within a sliding 6-month time frame while recognising that nominating one time frame would be extremely challenging. We would argue against a BR estimate based on the early time frames, as resistance due to antibiotic use might still persist and distort the estimate. However, we would also argue against a BR estimate based on the late time frames, as confidence intervals got wider at later time points and estimates less reliable. Since BR estimates were unstable and confidence intervals were wide, a sensitivity analysis using three different BR estimates (for time frames 3-8, 5-10 and 712 months) was conducted. BR estimates for $S$. pneumoniae calculated using samples from patients treated with $A F$ and $C D$ were slightly lower than the resistance rates reported by the European Antimicrobial Resistance Surveillance project (currently EARS-Net) co-ordinated by the European Centre for Disease Prevention and Control (http://ecdc.europa.eu/en/healthtopics/ antimicrobial-resistance-and-consumption/antimicrobial_resistance/EARS-Net; accessed 2 November 2016). BR estimates for $S$. pyogenes calculated using samples from patients treated with $\mathrm{AF}$ 

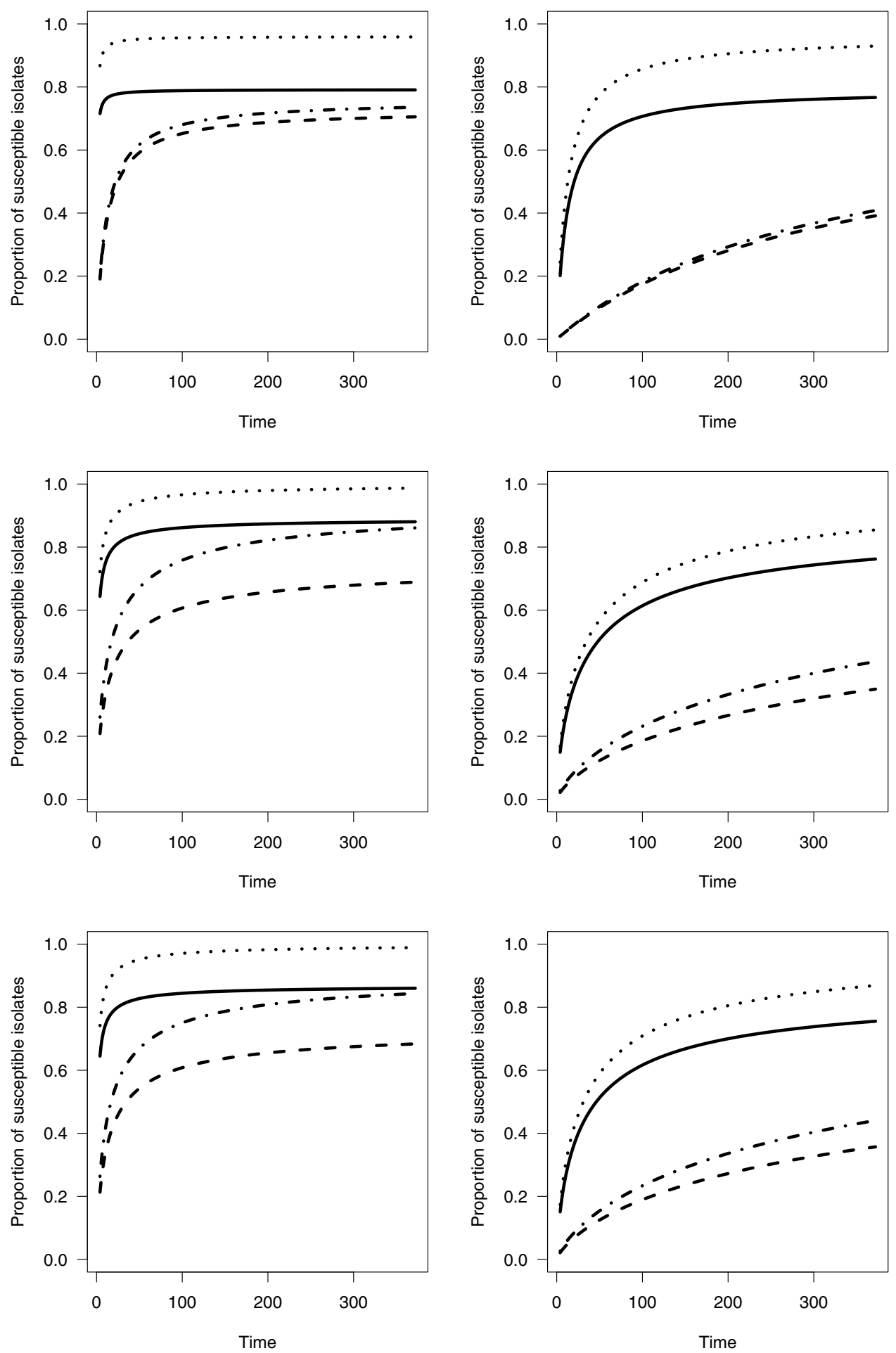

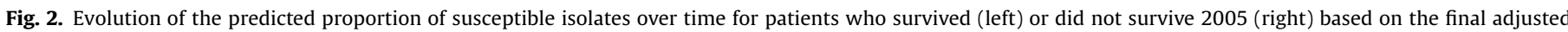

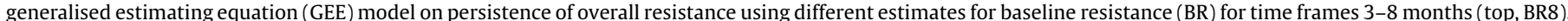

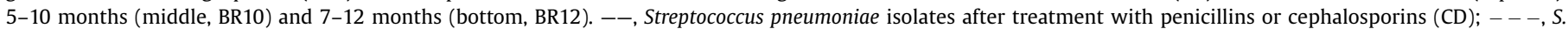

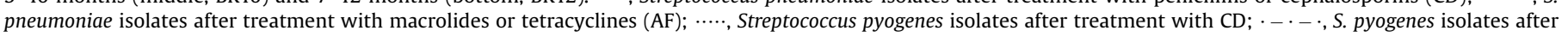
treatment with AF.

were slightly lower than the resistance rates reported by the Department of Medical Microbiology at the University of Antwerp. A possible explanation is that in contrast to the EARS-Net and the Department of Medical Microbiology at the University of Antwerp, in the current study BR was calculated based on a population of individuals who did not take any antibiotics during the last 3-8, 510 or 7-12 months (for BR8, BR10 and BR12, respectively). A limitation of our BR is that we can never be absolutely certain that patients did not consume antibiotics during the last months, e.g. leftovers from a treatment purchased longer than 12 months ago. Still, both the resistance rates obtained by EARS-Net and the Department of Medical Microbiology at the University of Antwerp and our BR might overestimate the resistance rate as mainly hospitalised patients and patients with treatment failure have 
Table 7

Parameter estimates for the generalised estimating equation (GEE) models on persistence of resistance for patients surviving 2005 using different estimates for baseline resistance (BR) $)^{\mathrm{a}}$ obtained by forward model building.

\begin{tabular}{lcrrr}
\hline \multirow{2}{*}{ Parameter } & \multicolumn{3}{l}{ Parameter estimate } & \\
\cline { 2 - 5 } & $\mathrm{BR}=0$ & \multicolumn{1}{l}{ BR8 } & \multicolumn{1}{c}{ BR10 } & \multicolumn{1}{c}{ BR12 } \\
\hline Intercept & -0.8259 & -2.4548 & -0.9597 & -0.7982 \\
Treatment AF & $-1.3199^{* * *}$ & -2.1939 & $-1.4629^{* *}$ & $-1.8904^{* *}$ \\
Bacteria PY & $1.6893^{* *}$ & 3.0169 & 0.8894 & 0.6218 \\
Log(time) & $0.5849^{* *}$ & $1.5567^{*}$ & $0.8512^{* *}$ & $0.8830^{* *}$ \\
Treatment AF $\times$ bacteria PY & $-1.1133^{*}$ & -1.9464 & -0.9061 & -0.3559 \\
\hline
\end{tabular}

AF, treatment with macrolides or tetracyclines; PY, Streptococcus pyogenes.

${ }^{*} P<0.05$.

${ }^{* *} P<0.01$.

a BR estimates for time frame 3-8 months (BR8), 5-10 months (BR10) and 712 months (BR12)

their resistance status tested. Nevertheless, we would recommend to report both an estimate of resistance rate with and without previous antibiotic treatment (BR) to get a more realistic picture of the true resistance rate in the general population.

The analysis showed that the odds of being susceptible increased significantly when the time between consumption and sampling increased for the respiratory streptococci studied, which confirms the conclusions reached by Chung et al. [8] and Malhotra-Kumar et al. [7] We also found that the rate of this

Table 8

Parameter estimates for the generalised estimating equation (GEE) models on persistence of resistance for patients surviving 2005 using different estimates for baseline resistance $(\mathrm{BR})^{\mathrm{a}}$ after final backward model reduction.

\begin{tabular}{llll}
\hline Parameter & \multicolumn{2}{l}{ Parameter estimate } & BR12 \\
\cline { 2 - 4 } & BR8 & BR10 & -0.5080 \\
\hline Intercept & -1.3733 & -0.5764 & $-2.1492^{* *}$ \\
Treatment AF & $-2.6361^{* *}$ & $-1.9909^{* *}$ & $0.9111^{* *}$ \\
Log(time) & $1.4817^{* *}$ & $0.8850^{* *}$ & 0
\end{tabular}

$\mathrm{AF}$, treatment with macrolides or tetracyclines; PY, Streptococcus pyogenes.

${ }^{*} P<0.05$

${ }^{* *} P<0.01$

a BR estimates for time frame 3-8 months (BR8), 5-10 months (BR10) and 712 months (BR12).

increase did not differ significantly between isolates from patients treated with $\mathrm{CD}$ or AF. This implies that when the proportion of susceptibility directly after treatment is comparable, persistence of resistance to these antimicrobial agents will likely not differ.

A reasonable assumption would be that the proportion of susceptibility directly after treatment equals $0 \%$. Malhotra-Kumar et al. [7], however, found $18 \%$ susceptible isolates after treatment with macrolides. Therefore, we did not make this assumption and allowed for different variables to influence the adjusted GEE model's intercept. The analysis including all patients showed that
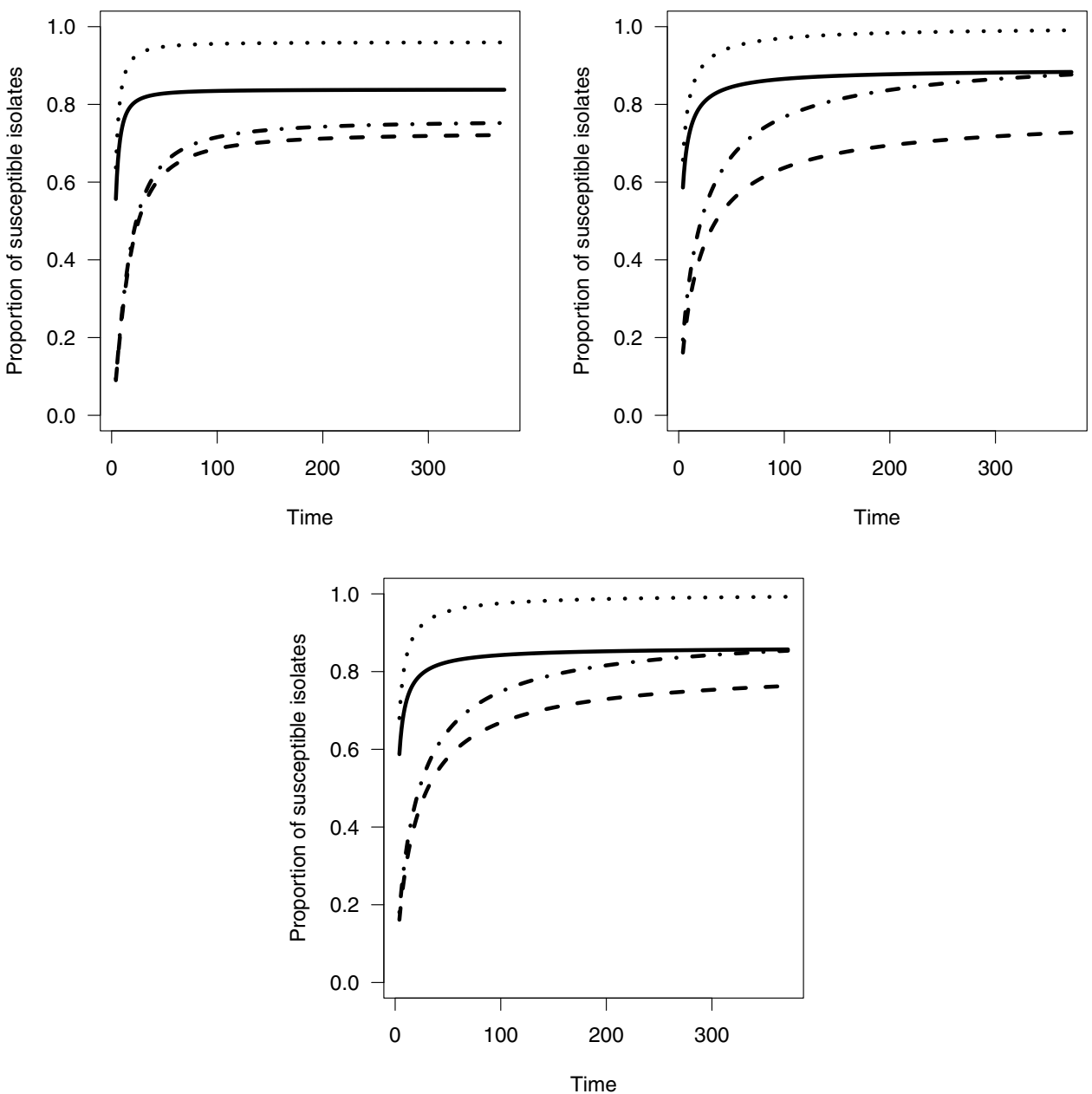

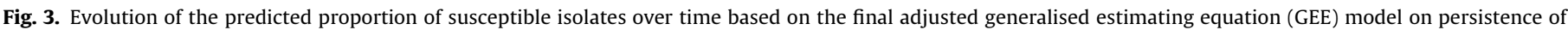

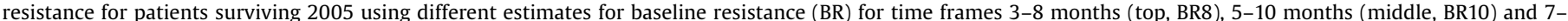

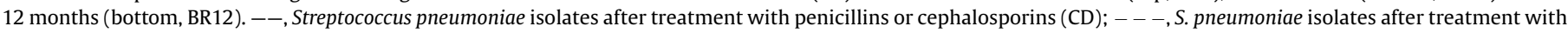
macrolides or tetracyclines (AF); $\cdots \cdot$, Streptococcus pyogenes isolates after treatment with $\mathrm{CD} ; \cdot-\cdot \cdot-\cdot$, . pyogenes isolates after treatment with $\mathrm{AF}$. 
Table 9

Number of days needed for the proportion of susceptible isolates to stabilise based on the final adjusted generalised estimating equation (GEE) model on persistence of resistance for patients surviving 2005 using different estimates for baseline resistance (BR). ${ }^{\mathrm{a}}$

\begin{tabular}{lrrr}
\hline Bug-drug combination & BR8 & BR10 & BR12 \\
\hline PY-CD & 44 & 71 & 66 \\
PN-CD & 44 & 71 & 66 \\
PY-AF & 125 & 195 & 194 \\
PN-AF & 125 & 195 & 194 \\
\hline
\end{tabular}

PY, Streptococcus pyogenes; PN, Streptococcus pneumoniae; CD, treatment with penicillins or cephalosporins; AF, treatment with macrolides or tetracyclines.

a BR estimates for time frame 3-8 months (BR8), 5-10 months (BR10) and 712 months (BR12).

the intercept differed significantly by treatment group and survival status. The analysis focusing on patients surviving 2005 revealed that the intercept differed significantly by treatment group.

The primary objective of this study was to assess whether the persistence of resistance to $\mathrm{CD}$ and AF differ. Whilst MalhotraKumar et al. [7] showed that persistence of resistance to macrolides lasts for up to 6 months, Chung et al. [8] indicated a much shorter persistence of resistance to penicillins. In this study, we found that the rate of increase in the odds of being susceptible did not differ between the two studied treatment groups, whilst the proportion of susceptible isolates directly after treatment was significantly lower for AF than for $\mathrm{CD}$. Therefore, it would take longer for the proportion of susceptible isolates to recover after treatment with $\mathrm{AF}$ than after treatment with $\mathrm{CD}$, hence confirming the differences found by Malhotra-Kumar et al. [7] and Chung et al. [8]. This difference in persistence of resistance is illustrated in Fig. 3 and Table 9. Note that this study focused on oral consumption of penicillins, cephalosporins, macrolides and tetracyclines. These findings cannot be extrapolated directly to parenteral consumption of the same antimicrobials [19].

The reported findings suggest the equivalence of the use of routinely collected data and prospective studies to answer such research questions. However, before drawing such a strong conclusion, there is need for additional validation of these findings for both the studied and other drug-bug combinations.

\section{Conclusions}

Resistance after treatment with AF persists longer than after treatment with $\mathrm{CD}$, which confirms the findings reported in prospective studies and suggest the equivalence of both designs in determining such differences. Our recommendation for future studies on persistence of resistance is to acknowledge the existence of BR and to use the adjusted GEE model rather than the regular GEE model.

\section{Competing interests}

None declared.

\section{Funding}

The work was supported by the Methusalem Financing Programme of the Flemish government and the IAP Research
Network P7/06 of the Belgian State (Belgian Science Policy). NH is supported by the University of Antwerp scientific chair in Evidence-Based Vaccinology, financed in 2009-16 by a gift from Pfizer. This study (IARG) was also supported by the Belgian National Council for the Promotion of Quality (Nationale Raad voor KwaliteitsPromotie; http://www.riziv.fgov.be/fr/inami/organes/ Pages/cnpq.aspx).

\section{Ethical approval}

Not required.

\section{References}

[1] French GL. Clinical impact and relevance of antibiotic resistance. Adv Drug Deliv Rev 2005;57:1514-27.

[2] Currie CJ, Berni E, Jenkins-Jones S, Poole CD, Ouwens M, Driessen S, et al Antibiotic treatment failure in four common infections in UK primary care 1991-2012: longitudinal analysis. BMJ 2014;349:g5493.

[3] Laxminarayan R, Duse A, Wattal C, Zaidi AKM, Wertheim HFL, Sumpradit N, et al. Antibiotic resistance-the need for global solutions. Lancet Infect Dis 2013;13:1057-98.

[4] Singer ME, Harding I, Jacobs MR, Jaffe DH. Impact of antimicrobial resistance on health outcomes in the out-patient treatment of adult communityacquired pneumonia: a probability model. J Antimicrob Chemother 2003;51:1269-82.

[5] Costelloe C, Metcalfe C, Lovering A, Mant D, Hay AD. Effect of antibiotic prescribing in primary care on antimicrobial resistance in individual patients: systematic review and meta-analysis. BMJ 2010;340:c2096.

[6] Goossens H, Ferech M, Vander Stichele R, Elseviers M, ESAC Project Group. Outpatient antibiotic use in Europe and association with resistance: a crossnational database study. Lancet 2005;365:579-87.

[7] Malhotra-Kumar S, Lammens C, Coenen S, Van Herck K, Goossens H. Effect of azithromycin and clarithromycin therapy on pharyngeal carriage of macrolide-resistant streptococci among healthy volunteers: a randomised, doubleblind, placebo-controlled study. Lancet 2007;369:482-90.

[8] Chung A, Perera R, Brueggemann AB, Elamin AE, Harnden A, Mayon-White R, et al. Effect of antibiotic prescribing on antibiotic resistance in individual children in primary care: prospective cohort study. BMJ 2007;335:429.

[9] Catry B, Hendrickx E, Preal R, Mertens R. Verband tussen antibioticaconsumptie en microbiële resistentie bij de individuele patient [Link between antibiotic consumption and antimicrobial resistance in the individual patient] Nationale Raad voor KwaliteitsPromotie; 2008. http://www.nsih.be/download/AB/multicentrstudienov2008.pdf; 2008 [Accessed 9 March 2016].

[10] WHO Collaborating Centre for Drug Statistics Methodology. Guidelines for ATC classification and DDD assignment 2011. Oslo, Norway: WHO Collaborating Centre for Drug Statistics Methodology; 2010 http://www.whocc.no/filearchive/publications/2011guidelines.pdf [Accessed 9 March 2016].

[11] Dever LA, Dermody TS. Mechanisms of bacterial resistance to antibiotics. Arch Intern Med 1991;151:886-95.

[12] Descheemaeker P. Macrolide resistance and erythromycin resistance determinants among Belgian Streptococcus pyogenes and Streptococcus pneumoniae isolates. J Antimicrob Chemother 2000;45:167-73.

[13] Molenberghs G, Verbeke G. Models for discrete longitudinal data. New York, NY: Springer; 2005.

[14] Agresti A. Categorical data analysis. Hoboken, NJ: John Wiley \& Sons; 2002.

[15] Liang K-Y, Zeger SL. Longitudinal data analysis using generalized linear models. Biometrika 1986;73:13-22.

[16] Putnam SD, Sanders JW, Tribble DR, Rockabrand DR, Riddle MS, Rozmajzl PJ, et al. Posttreatment changes in Escherichia coli antimicrobial susceptibility rates among diarrheic patients treated with ciprofloxacin. Antimicrob Agents Chemother 2005;49:2571-2.

[17] Raum E, Lietzau S, von Baum H, Marre R, Brenner H. Changes in Escherichia coli resistance patterns during and after antibiotic therapy: a longitudinal study among outpatients in Germany. Clin Microbiol Infect 2008;14:41-8.

[18] Shackcloth J, Williams L, Farrell DJ. Streptococcus pneumoniae and Streptococcus pyogenes isolated from a paediatric population in Great Britain and Ireland: the in vitro activity of telithromycin versus comparators. J Infect 2004;48:229-35.

[19] Zhang L, Huang Y, Zhou Y, Buckley T, Wang HH. Antibiotic administration routes significantly influence the levels of antibiotic resistance in gut microbiota. Antimicrob Agents Chemother 2013;57:3659-66. 